Discrete Comput Geom 29:41-59 (2003)

DOI: $10.1007 / \mathrm{s} 00454-002-2799-\mathrm{z}$

Geometry

\title{
The Combinatorial and Topological Complexity of a Single Cell*
}

\author{
Saugata Basu \\ School of Mathematics and College of Computing, Georgia Institute of Technology, \\ Atlanta, GA 30332, USA \\ saugata@math.gatech.edu
}

\begin{abstract}
The problem of bounding the combinatorial complexity of a single connected component (a single cell) of the complement of a set of $n$ geometric objects in $R^{k}$, each object of constant description complexity, is an important problem in computational geometry which has attracted much attention over the past decade. It has been conjectured that the combinatorial complexity of a single cell is bounded by a function much closer to $O\left(n^{k-1}\right)$ rather than $O\left(n^{k}\right)$ which is the bound for the combinatorial complexity of the whole arrangement. Until now, this was known to be true only for $k \leq 3$ and only for some special cases in higher dimensions.

A classic result in real algebraic geometry due to Oleinik and Petrovsky [15], Thom [18], and Milnor [14], bounds the topological complexity (the sum of the Betti numbers) of basic semi-algebraic sets. However, until now no better bounds were known if we restricted attention to a single connected component of a basic semi-algebraic set.

In this paper we show how these two problems are related. We prove a new bound on the sum of the Betti numbers of one connected component of a basic semi-algebraic set which is an improvement over the Oleinik-Petrovsky-Thom-Milnor bound. This also implies that the topological complexity of a single cell, measured by the sum of the Betti numbers, is bounded by $O\left(n^{k-1}\right)$. The techniques used for proving this topological result combined with those developed by Halperin and Sharir for the single cell problem in three dimensions allow us to prove a bound of $O\left(n^{k-1+\varepsilon}\right)$ on the combinatorial complexity of a single cell.

Finally, we show that under a certain natural geometric assumption on the objects (namely, that whenever they intersect, the intersection is robustly transversal) it is possible to prove a bound of $O\left(n^{k-1}\right)$ on the combinatorial complexity of a single cell.
\end{abstract}

* A preliminary version of this paper appeared in the Proceedings of the Symposium on the Foundations of Computer Science (FOCS), 1998. 


\section{Introduction}

Arrangements of a finite collection of geometric objects and their combinatorial and algorithmic properties have been a fundamental area of study in computational geometry. The most general setting considered in the computational geometry literature [1] is that of an arrangement, $A(\Sigma)$, of a set $\Sigma=\left\{S_{1}, \ldots, S_{n}\right\}$ of $n$ surface patches in $R^{k}$.

Each surface patch $S_{i}$ is a closed semi-algebraic set contained in a hypersurface $Z\left(Q_{i}\right)$ (the algebraic set defined by $Q_{i}=0$ ) and defined by a first-order quantifier-free formula involving a family of polynomials, $\left\{P_{i, 1}, \ldots, P_{i, r}\right\}$. We assume that the degrees of all the polynomials, $Q_{i}, P_{i, j}$, are bounded by $d$. Also, the parameters $r, d, k$ are constants independent of $n$ as is usual in the literature of computational geometry.

A cell is a maximal connected subset of the intersection of a fixed (possibly empty) subset of surface patches that avoids all other surface patches. Thus, a $k$-dimensional cell is a connected component of the complement of $S_{1}, \ldots, S_{n}$. The combinatorial complexity of the arrangement is the total number of cells of all dimensions. The combinatorial complexity of an $\ell$-dimensional cell $C$ is the number of cells of dimension less than $\ell$ which are contained in the relative boundary of $C$.

Usually, some additional geometric assumptions are made about the surface patches. In some of our theorems we impose the first or both of the following two conditions:

T1. Each surface patch is part of a smooth hypersurface and whenever surface patches intersect they do so transversally. Thus, the intersection of any $\ell$ surface patches is either empty or a $(k-\ell)$-dimensional manifold (with boundary). This also implies that no $k+1$ of the surface patches have a non-empty intersection.

T2. Suppose that the surface patches satisfy condition T1. For any $k$-dimensional cell $C$, the points of $\bar{C}$ (the closure of $C$ ) that are common to $k$ different surface patches are isolated. They are called the vertices of $C$. There exists a real constant, $b>0$, such that, if $x$ is a vertex of $C$ common to $k$ patches $S_{i_{1}}, \ldots, S_{i_{k}}$, then the unit normals $N_{1}, \ldots, N_{k}$ (chosen with any possible orientations) to these hypersurfaces at $p$ (see Fig. 1), span an ( $k$-dimensional) angle of at least $b$.

Note that this latter condition is a natural strengthening of the requirement that the surfaces meet transversally at the vertices. If they are required to meet transversally, then the solid angle will be positive. By requiring that the solid angle be at least $b$ we are imposing the extra condition that the transversality be robust.

One important problem that has remained open until now has been to bound the combinatorial complexity of a single $k$-dimensional cell in an arrangement of $n$ surface patches. Various special cases have been considered by researchers [16], [2], [17], [11]. In the special case, when each surface patch is a hyperplane, the combinatorial complexity of a single cell (which is a convex polytope with at most $n$ facets) is bounded by $n^{\lfloor k / 2\rfloor}$ by the upper bound theorem for simple polytopes [13]. In the more general situation the prevailing conjecture is that the combinatorial complexity of a single cell is bounded by $O\left(n^{k-1} \beta(n)\right)$ for some extremely slow growing function $\beta(n)$. This is known for $k=2$ (see [9]). The best known result for $k=3$ is due to Halperin and Sharir [11] who proved a bound of $O\left(n^{2+\varepsilon}\right)$ for every $\varepsilon>0$. In higher dimensions bounds close to $O\left(n^{k-1}\right)$ are known only for certain special cases. For instance, an $O\left(n^{k-1} \log n\right)$ bound was proved by Aronov and Sharir [2] (see also [17]) for the combinatorial com- 


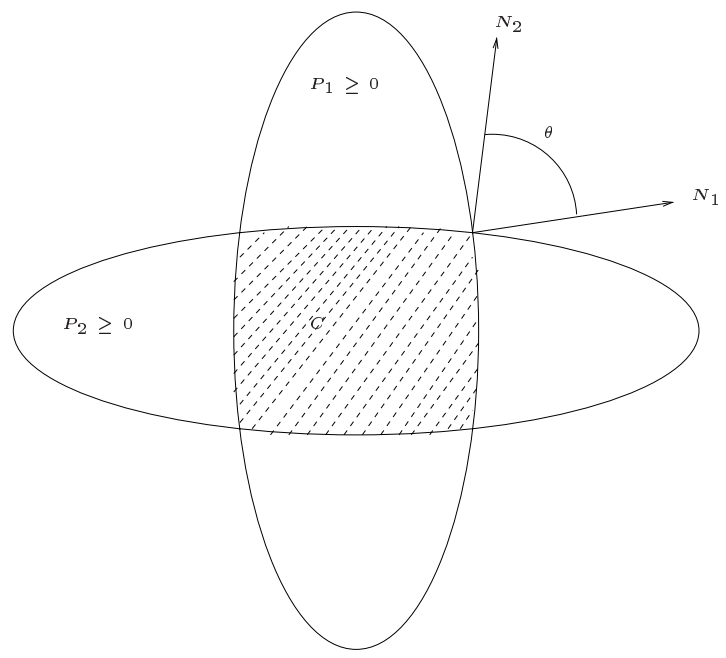

Fig. 1. The angle made by the normals is $\theta>b$.

plexity of a single cell in an arrangement of $n(k-1)$-simplices in $R^{k}$. These bounds are not known to extend or the proofs do not extend to general surface patches. The general problem was thus open in higher dimensions. Note also that using the lower bound construction given by Wiernik and Sharir [19] one can prove a lower bound of $\Omega\left(n^{k-1} \beta(n)\right)$, and hence an $O\left(n^{k-1}\right)$ bound is not possible. For a survey of the numerous algorithmic applications of these bounds in computational geometry and robotics see [1] and [10].

Another problem that has received considerable attention from researchers interested in real algebraic geometry, is to bound the topological complexity of semi-algebraic sets. A classical result in this area is due to Oleinik and Petrovsky [15], Thom [18], and Milnor [14], who independently proved that the sum of the Betti numbers, $\beta_{i}(S)$, of a basic closed semi-algebraic set $S \subset R^{k}$ defined by $P_{1} \geq 0, \ldots, P_{n} \geq 0$, with $\operatorname{deg}\left(P_{i}\right) \leq d, 1 \leq i \leq n$, is bounded by $O(n d)^{k}$. This bound was extended to arbitrary closed semi-algebraic sets in [3], where it is shown that the sum of the Betti numbers of a closed semi-algebraic set defined in terms of $n$ polynomials of degree $d$, which is contained in a real variety of dimension $k^{\prime}$ defined by a polynomial of degree at most $d$, is bounded by $n^{k^{\prime}} O(d)^{k}$.

The combinatorial part (the part depending on $n$ ) of these bounds are essentially tight as one can easily define a basic semi-algebraic set with $n$ polynomials and degrees bounded by $d$ which has $\Omega(n d)^{k}$ connected components. However, until now no attempt has been made to study the topological complexity of a single connected component of a basic semi-algebraic set. In analogy to the results bounding the combinatorial complexity of a single cell, one might conjecture that the sum of the Betti numbers of a single connected component of a basic semi-algebraic set is bounded by $n^{k-1} O(d)^{k}$. One cannot hope to do much better as it is quite easy to construct a basic semi-algebraic set defined in terms of $n$ polynomials of degree $d$ such that it has one connected component, the sum of whose Betti numbers is $\Omega(n d)^{k-1}$. 
Consider the following example: Let

$$
P_{i}=\left(X_{k}^{2}+L_{i, 1}^{2}\right) \cdots\left(X_{k}^{2}+L_{i,\lfloor d / 2\rfloor}^{2}\right)-\varepsilon,
$$

where the $L_{i j} \in R\left[X_{1}, \ldots, X_{k-1}\right]$ are generic linear polynomials and $\varepsilon>0$ and sufficiently small. The set $S$ defined by $P_{1} \geq 0, \ldots, P_{n} \geq 0$ has one connected component with $\beta_{1}(S)=\Omega(n d)^{k-1}$.

In this paper we prove the conjecture stated above. Moreover, we show how to approximate any collection of closed semi-algebraic sets in $R^{k}$, each of them having constant description complexity by semi-algebraic sets bounded by smooth hypersurfaces of small degrees. This enables one to apply tools from stratified Morse theory [8] directly to the problem of bounding the combinatorial complexity of a single cell. Together with the bound on the Betti numbers of a single connected component of a basic closed semialgebraic set, it also implies that the sum of the Betti numbers of a single cell is bounded by $O\left(n^{k-1}\right)$.

Using the techniques for proving the topological result described above, we are able to extend the Halperin-Sharir proof for the bound on the combinatorial complexity of a single cell in three dimensions to all higher dimensions. We provide the Morse theoretic tools needed to prove an $O\left(n^{k-1}\right)$ bound on the number of locally $X_{1}$-extreme vertices of a cell which was one of the obstructions to extending their proof to higher dimensions (see [1]) and show that the combinatorial complexity of a single cell in an arrangement of $n$ surface patches is bounded by $O\left(n^{k-1+\varepsilon}\right)$ for every $\varepsilon>0$. Further, under the additional assumption that the arrangement of surface patches satisfy conditions T1 and T2 we prove an $O\left(n^{k-1}\right)$ bound on the combinatorial complexity of a single cell.

\section{New Results}

First, we bound the topological complexity of a single connected component of a basic semi-algebraic set. This is the first complexity result which proves a bound which is better than the bound due to Oleinik and Petrovsky, Thom, and Milnor, for a single connected component of a basic semi-algebraic set.

Theorem 1. Let $C \subset R^{k}$ be a connected component of a basic semi-algebraic set defined by $P_{1} \geq 0, \ldots, P_{n} \geq 0$, with the degrees of the polynomials $P_{i}$ bounded by $d$. Then the sum of the Betti numbers of $C$ is bounded by $\left(\begin{array}{c}n \\ k-1\end{array}\right) O(d)^{k}$.

Actually, our technique proves the following stronger version of the above theorem.

Theorem 2. Let $C_{1}, \ldots, C_{m} \subset R^{k}$ be $m$ different connected components of a basic semi-algebraic set defined by $P_{1} \geq 0, \ldots, P_{n} \geq 0$, with the degrees of the polynomials $P_{i}$ bounded by $d$. Then $\sum_{i, j} \beta_{i}\left(C_{j}\right)$ is bounded by $m+\left(\begin{array}{c}n \\ k-1\end{array}\right) O(d)^{k}$.

For single cells we have the following results. 
Let $C$ be a $k$-dimensional cell in an arrangement of $n$ surface patches $S_{1}, \ldots, S_{n}$ in $R^{k}$. A corollary of Theorem 1 is the following:

Corollary 1. The sum of the Betti numbers of $C$ is bounded by $O\left(n^{k-1}\right)$.

Note that no general position assumption is required for the above corollary.

We are also interested in bounding the combinatorial complexity of $C$. In order to do this, it is enough to bound the number of vertices (the zero-dimensional cells) of $C$ (see [1]).

Theorem 3. Let $C$ be a k-dimensional cell in an arrangement of $n$ surface patches $S_{1}, \ldots, S_{n}$ in $R^{k}$ satisfying conditions $\mathrm{T} 1$ and $\mathrm{T} 2$. Then the number of vertices and hence the combinatorial complexity of $C$ is bounded by $O\left(n^{k-1}\right)$.

As before, we can prove a bound of $O\left(n^{k-1}+m\right)$ on the sum of the complexities of $m$ different cells. This has interesting consequences. For instance, this immediately gives us a bound of $O\left(n^{k-1}\right)$ on the combinatorial complexity of the zone of an algebraic variety of degree $d$ in an arrangement of $n$ surface patches in $R^{k}$.

Next, using the topological tools developed in this paper we are able to extend Halperin and Sharir's bound on the combinatorial complexity of a single cell in $R^{3}$ to all higher dimensions.

Theorem 4. Let $C$ be a k-dimensional cell in an arrangement of $n$ surface patches $S_{1}, \ldots, S_{n}$ in $R^{k}$, satisfying condition $\mathrm{T} 1$. Then the combinatorial complexity of $C$ is bounded by $O\left(n^{k-1+\varepsilon}\right)$ for every $\varepsilon>0$.

In Corollary 1 and Theorem 4 , the constants depend on the parameters $r, d, k$ used in the definition of the surface patches and also on $\varepsilon$. Additionally, in Theorem 3 , the constant also depends on the parameter $b$, bounding the cone angle at the vertices from below. The dependence on the degree $d$ is of the order of $2^{O(d)^{k}}$.

Remark 1. Using different techniques the author has recently proved new bounds on the individual Betti numbers of basic semi-algebraic sets [4] which implies Theorem 1. However, the technique used here is quite different and interesting in itself, as it is an essential ingredient in the proof of the new bound on the combinatorial complexity of a single cell.

\section{Mathematical Preliminaries}

In the following we sometimes perturb polynomials by various infinitesimals so that our geometric objects are semi-algebraic subsets of affine spaces over the field of Puiseux series in these infinitesimals. We write $R\langle\zeta\rangle$ for the real closed field of Puiseux series in $\zeta$ with coefficients in $R$ [5], [7]. The sign of such a Puiseux series agrees with the sign of 
the coefficient of the lowest degree term in $\zeta$. This order makes $\zeta$ positive and smaller than any positive element of $R$. The map eval ${ }_{\zeta}$ maps an element of $R\langle\zeta\rangle$ bounded over $R$ (one that has no negative powers of $\zeta$ ) to its constant term. An element of $R\langle\zeta\rangle$ is infinitesimal over $R$ if it is mapped by eval $\zeta$ to 0 . In particular, $\zeta$ is infinitesimal over $R$. If $S$ is a semi-algebraic subset of $R^{k}$ we denote by $S_{R\langle\zeta\rangle}$ the subset of $R\langle\zeta\rangle^{k}$ defined by the same equalities and inequalities that define $S$. Note that in the following, in all arguments involving infinitesimals, the infinitesimals can be replaced by sufficiently small positive real numbers. The main topological results we require are the two basic lemmas of stratified Morse theory which we now proceed to state.

For the rest of the paper we denote by $\pi$ the projection map onto the first co-ordinate. For any set $S \subset R^{k}$ we let $S_{x}$ (resp. $S_{\leq x}$ ) denote $S \cap \pi^{-1}(x)$ (resp. $S \cap \pi^{-1}((-\infty, x])$ ). A Whitney stratification of a space $X$ is a decomposition of $X$ into sub-manifolds called strata, which satisfy certain frontier conditions (see page 37 of [8]). In particular, given a compact set bounded by a smooth algebraic hypersurface, the boundary and the interior form a Whitney stratification.

Now, let $X$ be a compact Whitney stratified subset of $R^{k}$, and let $f$ be a restriction to $X$ of a smooth function. A critical point of $f$ is defined to be a critical point of the restriction of $f$ to any stratum, and a critical value of $f$ is the value of $f$ at a critical point. A function is called a Morse function if it has only non-degenerate critical points when restricted to each stratum, and all its critical values are distinct. (There is an additional non-degeneracy condition which states that the differential of $f$ at a critical point $p$ of a strata $S$ should not annihilate any limit of tangent spaces to a stratum other than $S$. However, in our situation this will always be true.)

The first fundamental result of stratified Morse theory is the following.

Theorem 5 [8]. As c varies in the open interval between two adjacent critical values, the topological type of $X \cap f^{-1}((-\infty, c])$ remains constant.

Stratified Morse theory actually gives a recipe for describing the topological change in $X \cap f^{-1}((-\infty, c])$ as $c$ crosses a critical value. This is given in terms of Morse data, which consists of a pair of topological spaces $(A, B), A \supset B$, with the property that as $c$ crosses the critical value $v=f(p)$, the change in $X \cap f^{-1}((-\infty, c])$ can be described by gluing in $A$ along $B$.

In stratified Morse theory the Morse data is presented as a product of two pairs, called the tangential Morse data and the normal Morse data. The notion of the product of pairs is the standard one in topology, namely,

$$
(A, B) \times\left(A^{\prime}, B^{\prime}\right)=\left(A \times A^{\prime}, A \times B^{\prime} \cup B \times A^{\prime}\right) .
$$

The tangential Morse data at a critical point $p$ is then given by $\left(B^{\lambda} \times B^{k-\lambda},\left(\partial B^{\lambda}\right) \times\right.$ $B^{k-\lambda}$ ) where $B^{k}$ is the closed $k$-dimensional disk, $\partial$ is the boundary map, and $\lambda$ is the index of the Hessian matrix (in any local co-ordinate system of the stratum containing $p$ in a neighborhood of $p$ ) of $f$ (restricted to the stratum containing $p$ ) at $p$.

Let $p=\left(p_{1}, \ldots, p_{k}\right)$ be a critical point in some $\ell$-dimensional stratum $S$ of a stratified subset $Z$ of $R^{k}$. 
Let $N^{\prime}$ be any $(k-\ell)$-dimensional hyperplane passing through the point $p$ which is transverse to $S$ which intersects the stratum $S$ locally at the single point $p$.

Let $B_{p}(\delta)$ be a closed ball centered at $p$ of radius $\delta$, for some sufficiently small $\delta$. Then the normal slice, $N(p)$, at the point $p$ is defined to be

$$
N(p)=N^{\prime} \cap Z \cap B_{p}(\delta) .
$$

Choose $\delta \gg \varepsilon^{\prime}>0$.

Let $\ell^{-}=N(p) \cap B_{p}(\delta) \cap \pi^{-1}\left(p_{1}-\varepsilon^{\prime}\right)$. The normal Morse data has the homotopy type of the pair (cone $\left.\left(\ell^{-}\right), \ell^{-}\right)$.

The following theorem measures the change in topology as we cross a critical value.

Theorem 6 [8, page 69]. Let $Z$ be a Whitney stratified space, let $f: Z \rightarrow R$ be a proper Morse function, and let $[a, b] \subset R$ be an interval which contains no critical values except for an isolated critical value $v \in(a, b)$ which corresponds to a critical point $p$ which lies in some stratum $S$ of $Z$. Let $\lambda$ be the Morse index of the critical point $p$. Then the space $Z_{\leq b}$ has the homotopy type of a space which is obtained from $Z_{\leq a}$ by attaching the pair $\left(B^{\lambda}, \partial B^{\lambda}\right) \times\left(\operatorname{cone}\left(\ell^{-}\right), \ell^{-}\right)$.

In our situation (because of transversality assumptions) the normal Morse data will be either empty or homotopic to (cone $\left.\left(B^{k-\ell-1}\right), B^{k-\ell-1}\right)$. In the first case, the Morse data is just the tangential Morse data. In the second case, there is no change in the homotopy type as the pair that is being added is contractible.

The following example should be helpful.

Example. Consider the (non-smooth) solid torus in Fig. 2 formed by rotating the shaded region enclosed by two curves, around the dotted line at the center. The resulting set has a natural stratification into one three-dimensional stratum, two two-dimensional strata, and two one-dimensional strata. There are four critical points $p_{1}, p_{2}, p_{3}, p_{4}$, with respect to the projection map onto the $X_{1}$ co-ordinate, all on the one-dimensional strata. The corresponding critical values are $c_{1}, c_{2}, c_{3}, c_{4}$. The critical points $p_{1}, p_{2}$ are of index 0 , and the critical points $p_{3}, p_{4}$ are of index 1 . The corresponding lower halffinks $\ell^{-}$ are shown in Fig. 2 for each critical point. Notice that the normal data (cone $\left.\left(\ell^{-}\right), \ell^{-}\right)$ is contractible for the critical points $p_{2}$ and $p_{4}$. Thus no change in the homotopy type occurs as the sweep plane crosses $c_{2}$ and $c_{4}$. At $c_{1}$ and $c_{3}$ a zero- and a one-dimensional ball get added, respectively. This shows that the solid torus has the same homotopy type as $S^{1}$, as one would expect.

We next bound the number of critical points of the projection map $\pi$ restricted to a stratum defined by a single polynomial equation.

Lemma 1. Let $Q \in R\left[X_{1}, \ldots, X_{k}\right]$ be a polynomial of degree at most $d$, and let $Z(Q) \subset R^{k}$ be a bounded smooth manifold of dimension $l \leq k$, such that the projection map onto the first co-ordinate, $\pi$, is a Morse function on $Z(Q)$. Then the number of critical points of $\pi$ restricted to $Z(Q)$ is bounded by $O(d)^{k}$. 


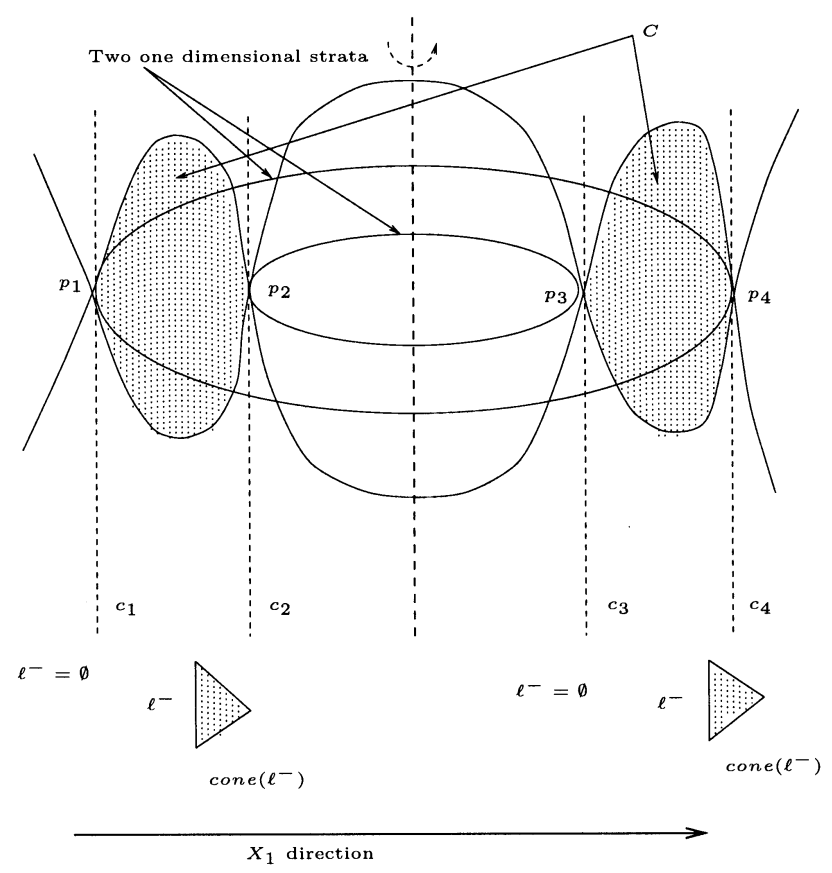

Fig. 2. Example of a non-smooth torus in $R^{3}$.

Proof. Consider the polynomial

$$
Q_{1}=(1-\zeta) Q^{2}-\zeta\left(X_{1}^{2 d+2}+\cdots+X_{k}^{2 d+2}+1\right),
$$

where $\zeta$ is an infinitesimal.

The algebraic set $Z\left(Q_{1}\right)$ is a smooth algebraic hypersurface of $R\langle\zeta\rangle^{k}$ on which $\pi$ (the projection of $\left(x_{1}, \ldots, x_{k}\right) \in R\langle\zeta\rangle^{k}$ to $x_{1} \in R\langle\zeta\rangle$ ) has $O(d)^{k}$ isolated critical points (see [6]).

Let $S^{\zeta}$ be the union of those connected components of the set defined by $Q_{1} \leq 0$ which intersect $Z(Q)$. Now, $Z(Q) \subset S^{\zeta}$. Moreover, for every $x \in R, Z(Q)_{\leq x}$ has the same homology groups as $S_{\leq x}^{\zeta}$ (see the proof of Proposition 4 in [3]).

Also, $S^{\zeta}$ is bounded by connected components of a smooth hypersurface $Z\left(Q_{1}\right)$, which has a finite number of critical points for the projection map onto the $X_{1}$ coordinate and these critical points are non-degenerate and have distinct $X_{1}$ co-ordinates.

We now claim that for every critical value of $Z(Q)$ there exists a critical value of $Z\left(Q_{1}\right)$ infinitesimally close to it. Since the number of critical values of $Z\left(Q_{1}\right)$ is bounded by $O(d)^{k}$ this would imply that the number of critical values and hence the number of critical points of $Z(Q)$ is bounded by $O(d)^{k}$.

We consider a sweep hyperplane orthogonal to the $X_{1}$ axis, and look at the sets $Z(Q)_{\leq x}, S_{\leq x}^{\zeta}$ as $x$ varies from $-\infty$ to $\infty$. From classical Morse theory we know that the homotopy type of $Z(Q)_{\leq x}$ changes as $x$ crosses a critical value. However, since $Z(Q)_{\leq x}$ and $S_{\leq x}^{\zeta}$ has the same homotopy type for every $x \in R$ this implies that the homotopy 
type of $S^{\zeta}$ has to change in an infinitesimal neighborhood of this critical value. However, since the homotopy type of $S_{\leq x}^{\zeta}$ changes only if $x$ crosses a critical value of $Z\left(Q_{1}\right)$, this proves that there exists a critical value of $Z\left(Q_{1}\right)$ in an infinitesimal neighborhood of every critical value of $Z(Q)$.

\section{Proofs of the Main Theorems}

We first prove a technical result which plays an important role in the proof of Theorem 3 . Let $\mathcal{P}=\left\{P_{1}, \ldots, P_{n}\right\} \subset R\left[X_{1}, \ldots, X_{k}\right]$ be a family of polynomials whose degrees are bounded by $d$.

Let $C$ be a connected component of the basic semi-algebraic set defined by $P_{1} \geq$ $0, \ldots, P_{n} \geq 0$.

We assume that the zero sets $Z\left(P_{i}\right)$ are smooth hypersurfaces such that the sign invariant sets of the polynomials $\left\{P_{1}, \ldots, P_{n}\right\}$ form a Whitney stratification of $R^{k}$ and that they satisfy conditions $\mathrm{T} 1$ and $\mathrm{T} 2$.

We prove the following proposition.

Proposition 1. If $C$ is defined as above and satisfies $\mathrm{T} 1$ and $\mathrm{T} 2$, then the number of vertices of $C$ is bounded by $\left(\begin{array}{c}n \\ k-1\end{array}\right) O(d)^{k}$.

Proof. We let $x$ vary from $-\infty$ to $+\infty$ and study what happens to $C_{\leq x}$.

First observe that the number of connected components of $C_{\leq x}$ changes only at finitely many points as $x$ varies, and each such $x$ is a critical value of a certain stratum of $C$, which is contained in an algebraic set of the form $P_{i_{1}}=\cdots=P_{i_{l}}=0$. Also, note that every vertex, being a zero-dimensional stratum, is a critical point.

Let $p$ be a vertex of $C$ and let $N_{1}, \ldots, N_{k}$ be the outer normals at $p$. We call $p$ a good vertex if and only if the the vector $(-1,0, \ldots, 0)$ which is normal to the hyperplane $X_{1}=0$ lies in the positive cone generated by the vectors $N_{1}, \ldots, N_{k}$ translated to the origin (see Fig. 3).

We also observe that if $p$ is a vertex of $C$ and $c=\pi(p)$, then the number of connected components of $C_{\leq x}$ changes (increases by one) as we cross $c$ from left to right if and only if $p$ is a good vertex. This follows from the fact that in a small neighborhood of $p$, the set $C$ can be very closely approximated by a convex cone bounded by the tangent hyperplanes at $p$ to the $k$ hypersurfaces incident on $p$. The total number of critical values coming from algebraic sets defined by fewer than $k$ polynomials is bounded by $\sum_{0 \leq \ell<k}\left(\begin{array}{c}n \\ \ell\end{array}\right)(O(d))^{k}=\left(\begin{array}{c}n \\ k-1\end{array}\right)(O(d))^{k}$ using Lemma 1. Also, for all large enough $x, C_{\leq x}$ is connected.

Thus, at every critical value corresponding to a good vertex a new connected component is born, no topological change occurs at critical values corresponding to vertices which are not good, and there are only $\left(\begin{array}{c}n \\ k-1\end{array}\right)(O(d))^{k}$ other critical values where the number of connected components can change. At these critical values only at most two connected components can join together. This allows us to bound the number of good vertices by $\left(\begin{array}{c}n \\ k-1\end{array}\right)(O(d))^{k}$.

Now, the outer normal cone at every vertex has an (solid) angle bounded from below by a constant $b$. Using a standard VC dimension argument and the theory of $\varepsilon$-nets [12], 


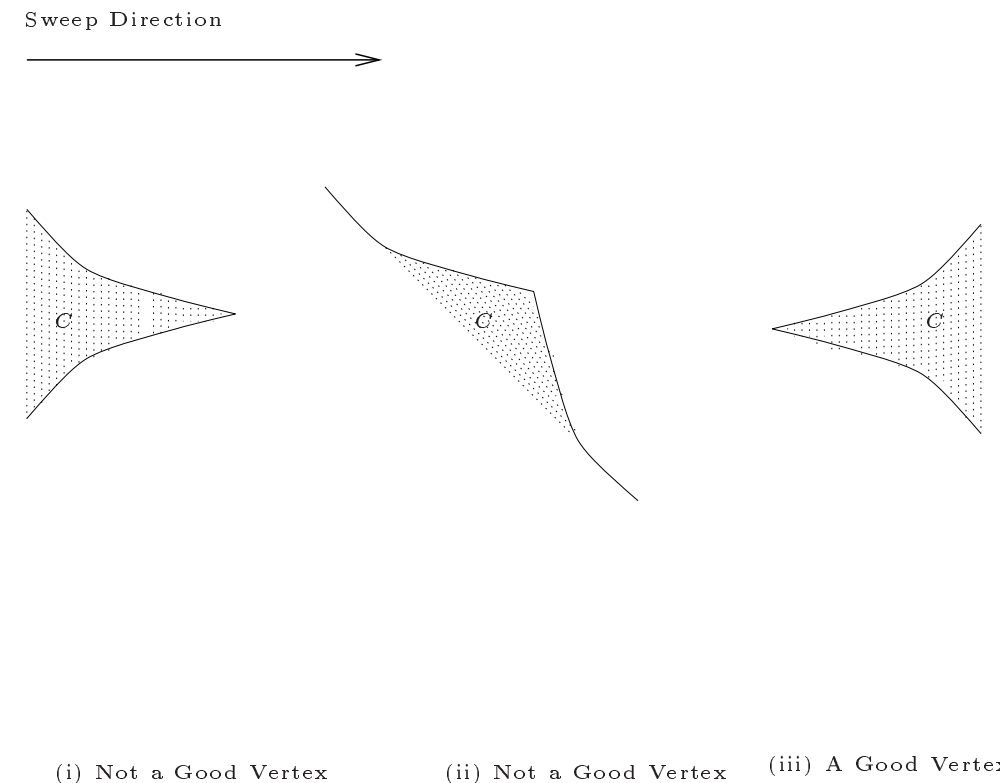

Fig. 3. Different kinds of vertices.

one can show that there exists a constant $c(b, k)$ and a set of $c(b, k)$ directions, such that every vertex is good for at least one direction. Thus, the total number of vertices is bounded by $c(b, k)\left(\begin{array}{c}n \\ k-1\end{array}\right) O(d)^{k}$.

Remark 2. Note that if we consider the number of vertices in any $m$ connected components, $C_{1}, \ldots, C_{m}$ instead of just $C$, the same argument bounds the number of good vertices by $m+\left(\begin{array}{c}n \\ k-1\end{array}\right)(O(d))^{k}$. This is so because at the end of the sweep we have $m$ components left rather than only one.

Remark 3. The bound on the number of good vertices in a particular direction is independent of the bound on the angle of the normal cones and thus does not require robust transversality (condition $\mathrm{T} 2$ ).

Remark 4. The proof is valid not only for a single connected component of a basic semi-algebraic set, but for a connected component of any semi-algebraic set defined as the intersection of $n$ semi-algebraic sets, each of which is bounded by a semi-algebraic hypersurface of bounded degree.

We next establish a connection between the single cell problem and the problem of bounding the number of vertices in a connected component of a set defined as the 
intersection of $n$ sets, each of which is bounded by a smooth hypersurface of bounded degree. We will then be in a position to use Proposition 1.

We first show how to replace each surface patch $S_{i}$ by a union of semi-algebraic sets, each of which is bounded by a smooth hypersurface. Moreover, their union contains $S_{i}$ and is infinitesimally close to it. For this we use a classical perturbation technique originally due to Milnor [14].

Let $S \subset R^{k}$ be a surface patch. For simplicity, we first assume that $S$ is a basic semialgebraic set defined by $P_{1} \geq 0, \ldots, P_{r} \geq 0$, and let $D$ be a connected component of $S$. We also assume that $D$ is bounded.

If the surface patch is contained in a variety $Z(Q)$, then we add $Q$ and $-Q$ to the set $\left\{P_{i}\right\}$.

Consider the set $S_{\varepsilon} \subset R\langle\varepsilon\rangle^{k}$ defined by $Q_{\varepsilon} \geq 0$, where

$$
Q_{\varepsilon}=\prod_{1 \leq i \leq r}\left((1-\varepsilon) P_{i}+\varepsilon\right)-\varepsilon^{r+1}\left(X_{1}^{2 d}+\cdots+X_{k}^{2 d}+1\right),
$$

where $2 d>\sum_{1 \leq i \leq r} \operatorname{deg}\left(P_{i}\right)$.

The set $S_{\varepsilon}$ contains $S$, and hence it has a connected component $D_{\varepsilon}$ which contains $D$ (see Fig. 4). Moreover, $D=\operatorname{eval}_{\varepsilon} D_{\varepsilon}$, and $D_{\varepsilon}$ is bounded by connected components of the smooth hypersurface $Z\left(Q_{\varepsilon}\right)$. For a proof of these facts see [3].

Thus, if all the surface patches $S_{1}, \ldots, S_{n}$ are basic semi-algebraic sets, then we can replace each connected component $D_{i, j}$ of $S_{i}$ by the corresponding set $D_{i, j, \varepsilon}$ defined as above. If $C$ is a connected component of the complement of $\bigcup S_{i}$, then there exists a connected component, $C_{\varepsilon} \subset R\langle\varepsilon\rangle^{k}$, of the complement of $\bigcup_{i, j} D_{i, j, \varepsilon}$ such that $C=$ $C_{\varepsilon} \cap R^{k}$. Moreover, $C_{\varepsilon}$ is a connected component of a set defined as the intersection of sets bounded by smooth hypersurfaces.

The next lemma shows that near smooth points of $S_{i}$ the unit normals to the approximating hypersurfaces $\partial D_{i, j, \varepsilon}$ closely approximate the normals to the original surface.

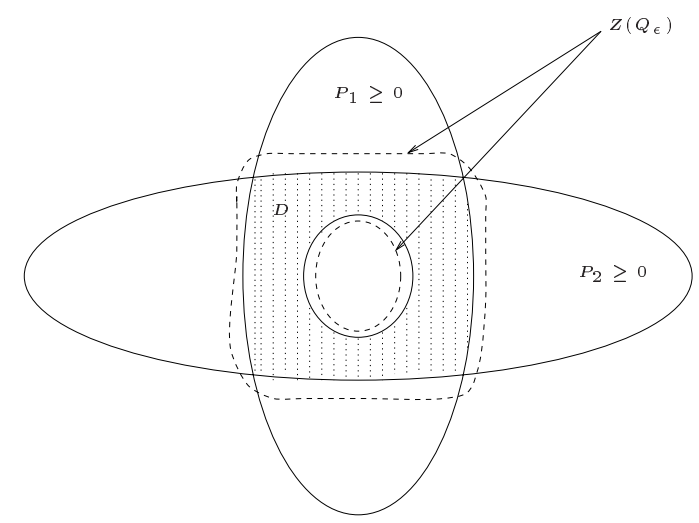

$$
D \text { is a connected component of } P_{1} \geq 0, P_{2} \geq 0 \text {. }
$$

Fig. 4. Approximating $D$ (the shaded region) by a connected component of $Q_{\varepsilon} \geq 0$. 
Lemma 2. For a smooth point $p$ of a connected component $D_{i, j}$ of a surface patch $S_{i}$, let $N_{p}$ be a unit normal at $p$. Then, inside any ball $B_{p}(\rho)$ around $p$ of infinitesimal radius $\rho$, and any point $q \in B_{p}(\rho) \cap \partial D_{i, j, \varepsilon}$, the unit normal $N_{q}$ is such that $1-\left|N_{p} \cdot N_{q}\right|$ is infinitesimal.

Proof. The proof uses Leibniz's rule to compute the partial derivatives of $Q_{\varepsilon}$ and is omitted.

It is clear that the number of vertices of $C_{\varepsilon}$ is at least the number of vertices of $C$. In order to see this, consider a vertex $p$ in $C$ which is the intersection of $k$ different surface patches, $S_{i_{1}}, \ldots, S_{i_{k}}$. Let the corresponding connected components be $D_{i_{1}, j_{1}}, \ldots, D_{i_{k}, j_{k}}$. Then in an infinitesimal neighborhood of $p$ a surface patch $S_{i_{1}}$ is sandwiched between two portions of the surface $\partial D_{i_{1}, j_{1}, \varepsilon}$. Then there is at least one vertex of $C_{\varepsilon}$ which is infinitesimally close to $p$ and which is in the intersection of the $k$ hypersurfaces, $\partial D_{i_{1}, j_{1}, \varepsilon}, \ldots, \partial D_{i_{k}, j_{k}, \varepsilon}$ (see Fig. 5).

Also, replacing the infinitesimal $\varepsilon$ by a variable $t$ we have that $C=\bigcup_{t>0} C_{t}$. Moreover, using Hardt's triviality theorem (see [7]) we have that for all sufficiently small $t>0$, the sets $C_{t}$ have the same homotopy type. Using the fact that the singular homology functor commutes with direct limit, we deduce that the homology groups of $C$ are isomorphic to those of $C_{t}$ for all sufficiently small $t>0$, and hence to those of $C_{\varepsilon}$.

This shows that we can replace the single cell $C$ by another semi-algebraic set $C_{\varepsilon}$ having the same homology groups, which is an intersection of sets bounded by smooth hypersurfaces, satisfying $\mathrm{T} 1$ and the number of vertices of $C_{\varepsilon}$ bounds the number of vertices of $C$.

We finally consider the case when the surfaces patches are not necessarily basic, but the surface patch $S_{i}$ is contained in $Z\left(Q_{i}\right)$ and is defined by some arbitrary Boolean

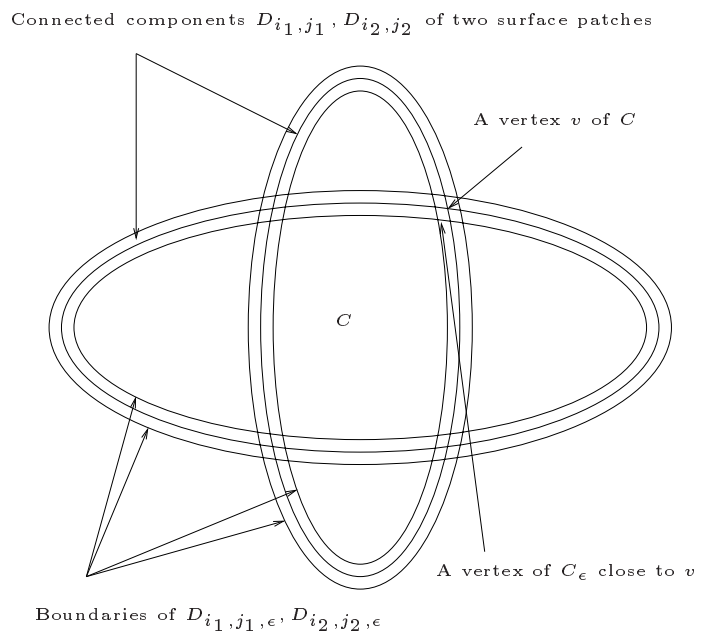

Fig. 5. Surface patches replaced by sets bounded by smooth hypersurfaces (in $R^{2}$ ). 
formula with atoms of the form $P_{i, j}\{\leq, \geq\} 0$. Since $S_{i}$ is closed it can be expressed as the union of $O\left(r^{k}\right)$ basic semi-algebraic sets (where $r$ is the number of polynomials appearing in the description of $S_{i}$ ). Thus, $S_{i}=\bigcup S_{i, j}$ where each $S_{i, j}$ is basic.

We replace each connected component of each of the basic semi-algebraic sets $S_{i, j}$ by sets bounded by smooth hypersurfaces as earlier.

We have proved the following proposition.

Proposition 2. Let $C$ be a connected component of the complement of the surface patches $S_{1}, \ldots, S_{n}$ satisfying condition $\mathrm{T} 1$ (resp. T1 and T2), intersected with a ball of sufficiently large radius. Then $C$ can be replaced by another semi-algebraic set, $C_{\varepsilon}$, having the same homology groups as $C$, which is an intersection of $O(n)$ semi-algebraic sets, each of which is bounded by a smooth hypersurface. Moreover, the hypersurfaces bounding these sets satisfy the conditions $\mathrm{T} 1$ (resp. T1 and T2). Further, the number of vertices of $C$ is $O(M)$ where $M$ is the number of vertices of $C_{\varepsilon}$.

The above proposition together with Proposition 1 (see Remark 3) proves Theorem 3.

\subsection{Bound on the Sum of the Betti Numbers of a Connected Component of a Basic Semi-Algebraic Set}

Let $C$ be a connected component of a basic semi-algebraic set $S$ defined by $P_{1} \geq$ $0, \ldots, P_{n} \geq 0$. By adding the additional polynomial inequality, $X_{1}^{2}+\cdots+X_{k}^{2}-\Omega \leq 0$, with sufficiently large $\Omega>0$, we can assume that $S$ is compact.

We now replace the set $S$ by a new set, $S^{+}(\bar{\varepsilon})$, defined by $P_{1} \geq-\varepsilon \varepsilon_{1}, \ldots, P_{n} \geq-\varepsilon \varepsilon_{n}$, where $\varepsilon_{1} \gg \varepsilon_{2} \gg \cdots \gg \varepsilon_{n} \gg \varepsilon>0$ are positive infinitesimals.

The following lemma appears in [3].

Lemma 3. $S^{+}(\bar{\varepsilon})$ has the same homology groups as $S$.

We also need the following lemma.

Lemma 4. The sets $Z\left(P_{i}+\varepsilon \varepsilon_{i}\right)$ are smooth hypersurfaces intersecting transversally. Moreover, the sign invariant sets of the family of polynomials, $\left\{P_{1}+\varepsilon \varepsilon_{1}, \ldots, P_{n}+\varepsilon \varepsilon_{n}\right\}$, give rise to a Whitney stratification of $R^{k}$.

Proof. It follows from the semi-algebraic version of Sard's lemma [7] that for almost all choices of $\varepsilon$ and $\varepsilon_{i}$ (that is, outside a semi-algebraic set of strictly lower dimension), $Z\left(P_{i}+\varepsilon \varepsilon_{i}\right)$ are smooth hypersurfaces intersecting transversally. Since the intersection of Whitney stratified sets intersecting transversally is also Whitney stratified the lemma follows.

Consider the connected component, $C^{+}(\bar{\varepsilon})$, of $S^{+}(\bar{\varepsilon})$ which contains $C$. By Lemma 3 , we have that $\beta_{i}(C)=\beta_{i}\left(C^{+}(\bar{\varepsilon})\right)$. Also, by a suitable change of co-ordinates we can assume that the projection map $\pi$ onto the first co-ordinate is a Morse function when restricted to each of the strata of $C^{+}(\bar{\varepsilon})$. 
We now look at the set $C^{+}(\bar{\varepsilon})_{\leq x}$ as $x$ goes from $-\infty$ to $+\infty$. Using Theorem 6 , it is easy to deduce that the sum of the Betti numbers of $C^{+}(\bar{\varepsilon})_{\leq x}$ increases by at most one as $x$ crosses a critical value corresponding to a critical point of a stratum of dimension $>0$. Since there are at most $\left(\begin{array}{c}n \\ k-1\end{array}\right) O(d)^{k}$ such critical points and we already know that there are only $\left(\begin{array}{c}n \\ k-1\end{array}\right) O(d)^{k}$ good vertices, this proves that the sum of the Betti numbers of $C^{+}(\bar{\varepsilon})$ and hence that of $C$ is bounded by $\left(\begin{array}{c}n \\ k-1\end{array}\right) O(d)^{k}$. This proves Theorem 1. Moreover, the above argument together with Remark 2 after the proof of Proposition 1 proves Theorem 2.

\section{Combinatorial Complexity of a Single Cell}

As remarked before, Halperin and Sharir [11] proved a bound of $O\left(n^{2+\varepsilon}\right)$ on the combinatorial complexity of a single cell in an arrangement of $n$ surface patches in $R^{3}$. The basic ingredients of their method was to prove two preliminary results needed to "bootstrap" the recurrences appearing in the analysis. These are:

1. a sharp bound on the number of locally $X_{1}$-extreme vertices of the cell $C$ (vertices whose $X_{1}$ co-ordinate is the smallest or largest in the closure of a connected component, of the intersection of $C$ with a small neighborhood of the vertex), and

2. a sharp bound on the number of vertices bounding "popular" faces of $C$ (faces that are adjacent to $C$ on all sides).

We first observe that from our bound on the number of good vertices we automatically derive an $O\left(n^{k-1}\right)$ bound on the number of $X_{1}$-extreme vertices thus taking care of the first item. This is because when we replace the surface patches by sets containing them which are bounded by smooth hypersurfaces, we create a good vertex of $C_{\varepsilon}$ in an infinitesimal neighborhood of every $X_{1}$-extreme vertex of $C$ (see Fig. 5).

Halperin and Sharir actually proved a bound on the number of incidences between vertices and popular faces. The cell itself (being a $k$-dimensional face) is popular. An $\ell$-dimensional face is popular if locally the cell $C$ lies in all its $2^{k-\ell}$ sides. We give formal definitions later. One difference between our argument and that in [11] is that we have an extra dimension in the recurrence. We bound the number of popular faces of a given dimension in terms of the number of popular faces of one lower dimension. The number of popular edges can be bounded by $O\left(n^{k-1}\right)$ using the bound on the number of $X_{1}$-extreme vertices, and this becomes the base case in the recurrence.

We also remark that in view of the fact that the set of surface patches changes in course of the inductive argument it is convenient to fix a point, say $Z \in R^{k}$, and always consider the single cell, $C_{Z}(\Sigma)$, containing this point, in whatever arrangement, $\Sigma$, we have in a particular step of the proof.

Following Halperin and Sharir, we call a vertex $v$ of an arrangement $A(\Sigma)$ an inner vertex if it is formed by the intersection of the relative interiors of $k$ distinct surfaces. In a small neighborhood of $v$ (assuming transversality of the intersection) the $k$ surfaces divide up the neighborhood into $2^{k}$ octants. A side $R$ is any one of these octants and we call the pair $(v, R)$ a 0 -border. We say that $(v, R)$ is a 0 -border of $C$ if the octant $R$ intersects $C$ (near $v$ ). 
We call a $(k-1)$-dimensional face popular if it borders $C$ on both sides. Similarly, we call any $\ell$-dimensional face popular if it borders $C$ on all its $2^{k-\ell}$ sides.

We let $\pi^{(\ell)}(n)$ denote the maximum number of incidences of vertices on $\ell$-dimensional popular faces. Notice that $\pi^{(k)}(n)$ is a bound on the combinatorial complexity of a single cell.

For a vertex $v$ which is the intersection of $k$ surface patches $S_{i_{1}}, S_{i_{2}}, \ldots, S_{i_{k}}$ we define the index of $v$ denoted by $j(v)$ to be the number of points of intersections of $S_{i_{1}}, S_{i_{2}}, \ldots, S_{i_{k}}$ to the right of $v$. Clearly, $0 \leq j(v) \leq N=O(d)^{k}$, where $d$ is the bound on the degrees of the polynomials defining the surface patches.

We define $\pi^{(\ell, j)}(\Sigma)$ to be the number of incidences of vertices of index at most $j$ on popular faces of dimension $\ell$, and $\pi^{(\ell, j)}(n)$ to be the maximum of $\pi^{(\ell, j)}(\Sigma)$ over all possible arrangements, $\Sigma$, of size $n$. Also, note that $\pi^{(\ell, N)}(n)=\pi^{(\ell)}(n)$.

We now obtain a recurrence on $\pi^{(\ell, j)}(n)$. Our method differs slightly from that of Halperin and Sharir, in that we have an extra index, $\ell$, in our recurrence which is the dimension of the popular faces that we are bounding. However, the analysis is still very similar in spirit.

Let $v$ be an inner vertex of a popular face $f$ of dimension $\ell$ of the cell $C$. Assume that $f$ lies in the intersection of $k-\ell$ surface patches $\sigma=\bigcap_{1 \leq i \leq k-\ell} S_{i}$ and let the vertex $v$ be defined as the intersection of the $k$ surface patches $S_{1}, \ldots, S_{k}$. (Note that if $\ell=k$, then the popular face is the cell $C$ itself and $\sigma=R^{k}$.)

For $k-\ell<i \leq k$, let $\gamma_{i}$ be the curve defined by $\bigcap_{1 \leq j \leq k, j \neq i} S_{j}$. Each $\gamma_{i}$ contains an edge $e_{i}$ incident on $v$ and $f$. Moreover, since $f$ is a popular face, and $e_{i}$ is incident on $f, 2^{k-\ell}$ of the $2^{k-1}$ sides of the edge $e_{i}$ lie locally in $C$.

Consider the curve $\gamma_{i}$. If $\gamma_{i}$ has another edge $e_{i}^{\prime}$ incident on another $\ell$-dimensional popular face $f^{\prime}$ contained in $\sigma$, and if $f$ and $f^{\prime}$ share a $(\ell-1)$-dimensional face $g$, then $g$ is a popular $(\ell-1)$-dimensional face of $C$. In this case we charge the incidence of $v$ on the popular face $f$ to the incidence of $v$ on the $(\ell-1)$-dimensional popular face $g$.

Otherwise, each of the curves $\gamma_{i}$ must contain a maximal relatively open $X_{1}$-monotone connected portion $\beta_{i}$, with one endpoint at $v$, such that at no point of $\beta_{i}$ do all the $2^{k-\ell}$ sides of $\beta_{i}$ consistent with the $2^{k-\ell}$ sides of $e_{i}$ lying locally in $C$, lie locally in $C$. Let $z_{i}$ denote the other endpoint of $\beta_{i}$. We will assume that at least one of the $\beta_{i}$ 's emanate in the positive $X_{1}$ direction and one in the negative $X_{1}$ direction as otherwise $v$ would be a $X_{1}$-extreme vertex in one of the $2^{k-\ell}$ sides of $f$ and we will bound the number of such vertices separately.

The analysis is divided into several cases.

Case (a). At least one of the curves $\beta_{i}$ ends at a point $z_{i}$ which is either an endpoint of the original intersection curve $\gamma_{i}$ or a point of local $X_{1}$-extremum on that curve. In either case we can charge $v$ to the point $z_{i}$ and note that each $z_{i}$ gets charged only a constant number of times in this process and the total number of such $z_{i}$ is $O\left(n^{k-1}\right)$. In what follows we assume that this situation does not hold, and hence $z_{i}$ is a vertex of an $\ell$-dimensional popular face of $C$.

Case (b). In this case we assume that $\beta_{i} \cup\left\{z_{i}\right\}$ is not intersected by the surface $S_{i}$. We define the popularity level of a point $w \in \sigma$ to be the smallest number of surfaces in $\Sigma$ whose removal makes $w$ lie in a popular $\ell$-dimensional cell contained in $\sigma$ in the resulting sub-arrangement. If $w$ is a vertex of $A(\Sigma)$ and incident to some face $f \subset \sigma$ 
we say that $(w, f)$ lies at a restricted popularity level $p(w, f)=m$ if by removing $m$ surfaces from $\Sigma$, none of which is incident to $w$, the face $f$ becomes a popular face of the cell containing $Z$ and if $m$ is the smallest number with this property.

Let $t$ denote the number of distinct surfaces of $\Sigma$ that intersect $\beta_{i} \cup\left\{z_{i}\right\}$. We fix some threshold parameter $\xi=\xi_{j}$ to be defined later and consider the following two sub-cases:

Case (b.i): $t \geq \xi$. In this case we charge $(v, f)$ to a block of $\xi$ points of intersection between $\beta_{i} \cup\left\{z_{i}\right\}$ and the surfaces of $\Sigma$ as follows. For each surface $S$ intersecting $\beta_{i} \cup\left\{z_{i}\right\}$ choose its point of intersection that lies nearest to $v$ along $\beta_{i}$. We obtain at least $\xi$ such designated points and we charge $(v, f)$ to the block of the first $\xi$ points. It is clear that each of these vertices can be charged no more than a constant number of times. Moreover, each charged vertex lies at a restricted popularity level at most $\xi$. We bound the number $M$ of pairs $\left(w, f^{\prime}\right)$ of inner vertices of $A(\Sigma)$ and incident $\ell$-dimensional faces $f^{\prime}$, that lie at restricted popularity level at most $\xi$; the number of pairs $(v, f)$ in the present sub-case being $O(M / \xi)$.

We choose a random sample $\mathcal{R}$ of $r=n / \xi$ surfaces of $\Sigma$. Let $w$ be an inner vertex of $A(\Sigma)$ at restricted level $p \leq \xi$, and let $\mathcal{Q}$ be a specific collection of $p$ surfaces, none incident to $w$, whose removal makes $w$ a vertex of a popular $\ell$-dimensional face. The probability that $w$ shows up as a vertex of $C_{Z}(\mathcal{R})$ is at least $\left(\begin{array}{c}n-p-k \\ r-k\end{array}\right) /\left(\begin{array}{c}n \\ r\end{array}\right)$. Thus, we have that

$$
\sum_{0 \leq p \leq \xi} \frac{\left(\begin{array}{c}
n-p-k \\
r-k
\end{array}\right)}{\left(\begin{array}{l}
n \\
r
\end{array}\right)} F_{p} \leq E\left[\pi^{(\ell)}(\mathcal{R})\right] \leq \pi^{(\ell)}(r),
$$

where $F_{p}$ is the number of pairs $\left(w, f^{\prime}\right)$ of $A(\Sigma)$ at restricted popularity level $p$ and $E[\cdot]$ denotes expectation. One can now easily verify that

$$
\sum_{0 \leq p \leq \xi} F_{p}=O\left(\xi^{k} \pi^{(\ell)}(n / \xi)\right)
$$

and so the number of pairs in this sub-case is $O\left(\xi^{k-1} \pi^{(\ell)}(n / \xi)\right)$.

Case (b.ii): $t<\xi$. In this case if these $t$ surfaces are removed from the arrangement, then $v$ becomes a vertex of a popular $(\ell-1)$-dimensional face of $C$. We can again use a random sampling argument by drawing a random sample $\mathcal{R}$ of $r=n / \xi$ surfaces of $\Sigma$.

Let $E^{\prime}[\mathcal{R}]$ be the expected number of vertices in $A(\mathcal{R})$ of index at most $j$ which are incident to popular $(\ell-1)$-dimensional faces of $C_{Z}(\mathcal{R})$. The probability that the vertex $v$ will show up as a vertex of such a popular face of $C_{Z}(\mathcal{R})$ is at least $\left(\begin{array}{c}n-t-k \\ r-k\end{array}\right) /\left(\begin{array}{c}n \\ r\end{array}\right)$. If $G_{t}$ is the number of pairs in the full arrangement that falls into the present sub-case, we have that

$$
\sum_{0 \leq t \leq \xi} \frac{\left(\begin{array}{c}
n-t-k \\
r-k
\end{array}\right)}{\left(\begin{array}{c}
n \\
r
\end{array}\right)} G_{t} \leq E^{\prime}[\mathcal{R}] \leq \pi^{(\ell-1, j)}(r) .
$$

Again, this implies that

$$
\sum_{0 \leq t \leq \xi} G_{t}=O\left(\xi^{k} \pi^{(\ell-1, j)}(n / \xi)\right)
$$


Case (c). In the remaining case (which can only happen if $j(v)>0$ ), the surface $S_{i}$ intersects $\beta_{i} \cup\left\{z_{i}\right\}$ in at least one point $w$ (if there are several such points take $w$ to be the farthest one from $v$ along $\beta_{i}$ ). Again, let $t$ denote the number of distinct surfaces of $\Sigma$, excluding $S_{i}$, that intersect $\beta_{i} \cup\left\{z_{i}\right\}$. We have two sub-cases:

Case (c.i): $t \geq \xi$. This case is similar to case (b.i) earlier and we charge $(v, f)$ to a block of $\xi$ vertices as in (b.i). Using a similar argument as in (b.i) one can show that the number of pairs in this sub-case is restricted to $O\left(\xi^{k-1} \pi^{(l)}(n / \xi)\right)$.

Case (c.ii): $t<\xi$. In this case if we remove these $t$ surfaces (without removing $S_{i}$ ) the point $w$ becomes a vertex of an $\ell$-dimensional popular face $f^{\prime}$ of the resulting subarrangement. In this case we charge $(v, f)$ to the pair $\left(w, f^{\prime}\right)$. Clearly, each pair $\left(w, f^{\prime}\right)$ is charged only a constant number of times in this way.

We next estimate the number of such pairs $\left(w, f^{\prime}\right)$. We again draw a random sample $\mathcal{R}$ of $r=n / \xi$ surfaces of $\Sigma$. Let $E^{\prime \prime}[\mathcal{R}]$ be the expected number of pairs $\left(w, f^{\prime}\right)$ of $C_{Z}(\mathcal{R})$ such that $w$ has index $\leq j-1$. If $H_{t}$ is the number of pairs $(v, f)$ that fall in the present sub-case, using similar arguments as before we get

$$
\sum_{0 \leq t \leq \xi} \frac{\left(\begin{array}{c}
n-t-k \\
r-k
\end{array}\right)}{\left(\begin{array}{l}
n \\
r
\end{array}\right)} H_{t} \leq E^{\prime \prime}[\mathcal{R}] \leq \pi^{(\ell, j-1)}(r)
$$

This implies that

$$
\sum_{0 \leq t \leq \xi} H_{t}=O\left(\xi^{k} \pi^{(\ell, j-1)}(n / \xi)\right) .
$$

Summing all the cases we obtain the following recurrence on $\pi^{(\ell, j)}(n)$ :

$$
\begin{aligned}
\pi^{(\ell, j)}(n)=O & \left(\pi^{(\ell-1, j)}(n)+\xi^{k-1} \pi^{(\ell)}(n / \xi)+\xi^{k} \pi^{(\ell-1, j)}(n / \xi)\right. \\
& \left.+\xi^{k} \pi^{(\ell, j-1)}(n / \xi)+n^{k-1}\right) .
\end{aligned}
$$

Also, note that $\pi^{(0, j)}(n)=O\left(n^{k-1}\right)$ for $0 \leq j \leq N$. This is because a popular vertex of $C$ must be locally extreme for at least one of the $2^{k}$ sides it is incident on, and the number of such vertices is bounded by $O\left(n^{k-1}\right)$. This also serves as the base case for our induction.

These recurrences yield the desired bound of $O\left(n^{k-1+\varepsilon}\right)$ on $\pi^{(k)}(n)$ and hence on the combinatorial complexity of a single cell.

\subsection{Solving the Recurrence}

We follow a similar scheme as in [11] with minor modifications. Fix some $\varepsilon>0$. We claim that $\pi^{(\ell, j)}(n) \leq B_{\ell, j} n^{k-1+\varepsilon}$, for $0 \leq \ell \leq k, 0 \leq j \leq N$, where the constants $B_{\ell, j}$ depend on $\varepsilon, \ell, j$ and on the parameters $r, d, k$ (see Section 1) used in the definition of the surface patches.

The proof is by induction on $\ell$ and $n$. Recall that $\pi^{(0, j)}(n)=O\left(n^{k-1}\right)$ for all $j$, and this serves as the base case for our induction. 
We use a different parameter $\xi_{\ell, j}$ for each pair $\ell, j$ in the recurrence. Thus, rewriting the recurrence equations, we have

$$
\begin{gathered}
\pi^{(\ell, j)}(n) \leq c\left(\pi^{(\ell-1, j)}(n)+\xi_{\ell, j}^{k-1} \pi^{(\ell)}\left(n / \xi_{\ell, j}\right)+\xi_{\ell, j}^{k} \pi^{(\ell-1, j)}\left(n / \xi_{\ell, j}\right)\right. \\
\left.+\xi_{\ell, j}^{k} \pi^{(\ell, j-1)}\left(n / \xi_{\ell, j}\right)+n^{k-1}\right),
\end{gathered}
$$

for appropriate constants $c, \xi_{\ell, j}$. We assume that $c>1$, that $\xi_{0,0}$ is sufficiently large, and put $\xi_{\ell, j}=\xi_{0,0}^{\varepsilon^{\ell+j}}$. Also, by choosing the $B_{\ell, j}$ 's sufficiently large we can assume that the claimed bounds hold for all $n \leq \xi_{0,0}$.

We choose $B_{0,0}>2 c \xi_{0,0}^{1-\varepsilon}$ and $B_{\ell, j}=\left((\ell+j+1)(3 c)^{\ell+j} / \xi_{\ell, j}^{\varepsilon}\right) \xi_{0,0}^{\varepsilon} B_{0,0}$, and require that $\xi_{0,0}$ be sufficiently large so that $\xi_{0,0}^{\varepsilon^{k+N+1}}>2 c(k+N+1)(3 c)^{k+N}$.

Applying the inductive hypothesis on the right side of the above recurrence we see that the claimed bounds will hold if the following inequalities are satisfied:

$$
c B_{\ell-1, j}+\frac{c B_{k, N}}{\xi_{\ell, j}^{\varepsilon}}+c\left(B_{\ell-1, j}+B_{\ell, j-1}\right) \xi_{\ell, j}^{1-\varepsilon}+\frac{c}{\xi_{0,0}^{\varepsilon}} \leq B^{\ell, j},
$$

for $0 \leq \ell \leq k, 0 \leq j, \leq N$.

Note that $B_{\ell-1, j}=B_{\ell, j-1}$, and hence the above inequality is implied by

$$
\begin{aligned}
& \frac{c(k+N+1)(3 c)^{k+N} \xi_{0,0}^{\varepsilon} B_{0,0}}{}+\frac{3 c(\ell+j)(3 c)^{\ell+j-1} \xi_{0,0}^{\varepsilon} B_{0,0}}{\xi_{0,0}^{\varepsilon^{\ell+N+1}} \xi_{0,0}^{\varepsilon^{\ell+j+1}}}+\frac{c}{\xi_{0,0}^{\varepsilon}} \\
& \leq \frac{(\ell+j+1)(3 c)^{\ell+j} \xi_{0,0}^{\varepsilon} B_{0,0}}{\xi_{0,0}^{\ell^{\ell+j+1}}} .
\end{aligned}
$$

Using the fact that, $\xi_{0,0}^{\varepsilon^{k+N+1}}>2 c(k+N+1)(3 c)^{k+N}$, the last inequality is seen to be implied by

$$
\frac{c}{\varepsilon_{0,0}^{\varepsilon}} \leq \frac{\left((3 c)^{\ell+j}-\frac{1}{2}\right) \xi_{0,0}^{\varepsilon} B_{0,0}}{\xi_{0,0}^{\varepsilon+j+1}}
$$

or by

$$
\frac{c \xi_{\ell, j}^{1+\varepsilon}}{\xi_{0,0}^{\varepsilon}} \leq\left((3 c)^{\ell+j}-\frac{1}{2}\right) \xi_{0,0}^{\varepsilon} B_{0,0} .
$$

However, when $\ell+j$ increases, the left-hand side decreases while the right-hand side increases. Hence, it suffices to verify the last inequality for $\ell=j=0$, which holds by our choice of $B_{0,0}$. This completes the induction.

\section{Acknowledgments}

I thank Ricky Pollack, Boris Aronov, Micha Sharir, and Marie-Francoise Roy for discussions and helpful comments. 


\section{References}

1. P.K. Agarwal, M. Sharir, Arrangements and their applications, in Handbook of Computational Geometry, J.R. Sack, J. Urrutia (eds.), Elsevier, Amsterdam, 2000, pp. 49-120.

2. B. Aronov, M. Sharir, Castles in the air revisited, Discrete and Computational Geometry, 12 (1994), 119-150.

3. S. Basu, On bounding the Betti numbers and computing the Euler characteristics of semi-algebraic sets, Discrete \& Computational Geometry, 22 (1999), 1-18.

4. S. Basu, On different bounds on different Betti numbers, Discrete \& Computational Geometry, to appear. (Preliminary version in Proceedings of the ACM Symposium on Computational Geometry, pp. 288-292, 2001.) Available at www. math.gatech.edu/ saugata/journalsocg $01 . p s$.

5. S. Basu, R. Pollack, M.-F. Roy, On the combinatorial and algebraic complexity of Quantifier Elimination, Journal of the Association for Computing Machinery, 43 (1996), 1002-1045.

6. S. Basu, R. Pollack, M.-F. Roy, Computing roadmaps of semi-algebraic sets on a variety, Journal of the American Mathematical Society, 13 (2000), 55-82.

7. J. Bochnak, M. Coste, M.-F. Roy, Géométrie algébrique réelle, Springer-Verlag, Berlin, 1987.

8. M. Goresky, R. MacPherson, Stratified Morse Theory, Springer-Verlag, New York, 1987.

9. L.J. Guibas, M. Sharir, S. Sifrony, On the general motion planning problem with two degrees of freedom, Discrete \& Computational Geometry, 4 (1989), 491-521.

10. D. Halperin, Arrangements, in Handbook of Discrete and Computational Geometry, J. O'Rourke, J.E. Goodman (eds.), CRC Press, Boca Raton, FL, 1997, pp. 389-412.

11. D. Halperin, M. Sharir, Almost tight upper bounds for the single cell and zone problems in three dimensions, Discrete \& Computational Geometry, 14 (1995), 385-410.

12. D. Haussler, E. Welzl, Epsilon-nets and simplex range queries, Discrete \& Computational Geometry, 2 (1987), 127-151.

13. P. McMullen, The maximum number of faces of a convex polytope, Mathematika, 17 (1970), 179-184.

14. J. Milnor, On the Betti numbers of real varieties, Proceedings of the American Mathematical Society, 15 (1964), 275-280.

15. O.A. Oleinik, I.B. Petrovsky, On the topology of real algebraic surfaces, Izvestiya Akademii Nauk SSSR, 13 (1949), 389-402.

16. J. Pach, M. Sharir, The upper envelope of piecewise linear functions and the boundary of a region enclosed by convex plates: combinatorial analysis, Discrete \& Computational Geometry, 4 (1989), 291-309.

17. B. Tagansky, A new technique for analyzing substructures in arrangements of piecewise linear surfaces, Discrete \& Computational Geometry, 16 (1996), 455-479.

18. R. Thom, Sur l'homologie des varietes algebriques reelles, in Differential and Combinatorial Topology, S.S. Cairns (ed.), Princeton University Press, Princeton, NJ, 1965, pp. 255-265.

19. A. Wiernik, M. Sharir, Planar realization of nonlinear Davenport-Schinzel sequences by segments, Discrete \& Computational Geometry, 3 (1988), 15-47.

Received December 28, 2000, and in revised form July 9, 2002. Online publication November 14, 2002. 